\title{
Freie Aminosäuren und verwandte Verbin- dungen in der Muskulatur der Chimäre sowie einiger Elasmobranchier und Evertebraten
}

\author{
Von Heinz Schaefer \\ Aus dem Institut für Meereskunde der Universität Kiel \\ und der Biologischen Anstalt Helgoland
}

Über freie Aminosäuren bei Elasmobranchiern liegen sehr wenige Angaben vor. Nach Shewan (1953), der ihr Auftreten bei Raja batis, Acanthias vulgaris und Mustelus vulgaris säulenchromatographisch analysierte, unterscheiden sich diese Elasmobranchier von den Gadiden qualitativ durch das Vorkommen von Betain und Sarcosin, quantitativ durch stärkeres Auftreten von Trimethylaminoxyd, während bei den Gadiden die basischen Komponenten stärker nachweisbar sind. Der Katzenhai Scyliorhinus canis besitzt nach den papierchromatographischen Untersuchungen von RANKE (1959) größere Mengen von Arginin, Prolin und Asparaginsäure als die von ihm bearbeiteten Knochenfische.

Über Chimaera fehlen entsprechende Analysen.

Über einzelne freie Aminosäuren bzw. über deren Gesamtmuster bei marinen Evertebraten wurde von mehreren Autoren berichtet (vgl. Simpson et al. 1959). Eine Zusammenstellung der Literatur muß einer späteren Übersicht überlassen bleiben.

Die vorliegende Arbeit beschäftigt sich ebenfalls mit dem Muster der freien Aminosäuren in der Muskulatur mariner Tiere, von denen in dieser Hinsicht zum größten Teil noch nichts bekannt ist. Die Untersuchungen sollen dazu dienen, weitere chemische Charakteristika der systematisch so unterschiedlichen Arten herauszustellen.

\section{Material und Methodik}

Die Muskulatur der untersuchten Tiere wurde gleich nach dem Fang an Bord von FFS „Anton Dohrn“ zu alkoholischen Extrakten verarbeitet. Die Untersuchungsmethoden, einige Reaktionen der noch nicht identifizierten Sub-

Deutscher Beitrag zum Polarfrontprogramm des Internationalen Geophysikalischen Jahres 1958

Der Deutschen Forschungsgemeinschaft danke ich ergebenst für die Gewährung eines Stipendiums und einer Sachbeihilfe. 
stanzen und die papierchromatographische Lage der meisten dieser Verbindungen wurden in einer früheren Arbeit beschrieben (Schaefer 1962). Die Position der übrigen unbekannten Substanzen auf den Papierchromatogrammen (Lösungsmittel Butanol : Eisessig: Wasser 4:1:1 and Pyridin : Amylalkohol : Wasser 7,5:7:6) ist wie folgt: „3" unter Tryptophan auf der Höhe von Glycin; „9“ zwischen $\alpha$-Aminobuttersäure und Valin; „15“ unter Valin auf der Höhe von Threonin.

Folgende Muskelpartien wurden untersucht:
Elasmobranchier
Tellina, Neptunia
Rumpfmuskulatur
r'andalus
Fußmuskulatur
Gonatus
Abdominalmuskulatur
Ringmuskel und Tentakel

Von den Muskeln einiger Evertebraten wurde kein Frischgewicht bestimmt, die darauf zu beziehenden Stickstoffwerte entfallen daher.

\section{Ergebnisse}

Die Positionen der Fangplätze und hydrographische Angaben sind in Tabelle 1 wiedergegeben.

Der Gehalt an Gesamtstickstoff und Reststickstoff in den alkoholischen Muskelextrakten der Elasmobranchier und der Chimäre (Tabelle 2) ist etwa 3-4mal so hoch wie der der Knochenfische (vgl. Schaefer 1962). Der Gehalt an Aminostickstoff liegt mit Ausnahme des gefleckten Rochens nicht sehr viel höher als der von Knochenfischen mit hohen Aminostickstoff-Konzentrationen. Das Verhältnis Aminostickstoff zu Gesamtstickstoff entspricht dem der meisten Knochenfische. Der Reststickstoff muß also durch andere stickstoffhaltige Substanzen verursacht sein, die unter den vorliegenden Arbeitsbedingungen nicht die van-Slyke-Reaktion ergeben. Vermutlich spielt hierbei der Harnstoff, eventuell auch Betain und Trimethylaminoxyd eine große Rolle. Bereits STÄDELER und Frerichs (1858) fanden sehr große Harnstoffmengen in der Elasmo-

Tabelle 1

Hydrographische Angaben zu den Fangstationen

\begin{tabular}{|c|c|c|c|c|c|c|c|}
\hline Station & Position & & & Datum & Fangtiefe & Temperatur & Salzgehalt \\
\hline 2233 & Kanal & $\begin{array}{r}50^{0} 25^{\prime} \\
0^{0} 12^{\prime}\end{array}$ & $\begin{array}{l}\mathrm{N} \\
\mathrm{W}\end{array}$ & 1. 3. & $49-56 \mathrm{~m}$ & 8,48 & $35,07 \%$ \\
\hline 2234 & Kanal & $\begin{array}{l}49^{0} 58^{\prime} \\
04^{0} 02^{\prime}\end{array}$ & $\begin{array}{l}\mathrm{N} \\
\mathrm{W}\end{array}$ & 2. 3 . & 72 & 9,62 & 35,10 \\
\hline 2301 & südl. Island & $\begin{array}{l}63^{0} 05^{\prime} \\
23^{0} 57^{\prime}\end{array}$ & $\begin{array}{l}\mathrm{N} \\
\mathrm{W}\end{array}$ & 28.3. & $430-460$ & 6,45 & 35,19 \\
\hline 2477 & Nordsee & $\begin{array}{l}54^{0} 58^{\prime} \\
05^{\circ} 40^{\prime}\end{array}$ & $\begin{array}{l}\mathrm{N} \\
\mathrm{O}\end{array}$ & 6. 8. & 41 & & \\
\hline 2478 & Nordsee & $\begin{array}{l}57^{0} 42^{\prime} \\
0^{0} 12^{\prime}\end{array}$ & $\begin{array}{l}\mathrm{N} \\
\mathrm{W}\end{array}$ & 7. 8. & 95 & & \\
\hline 2620 & $\begin{array}{l}\text { Cap Farvel } \\
\text { (Südgrönland) }\end{array}$ & $\begin{array}{l}59^{0} 41^{\prime} \\
44^{0} 12^{\prime}\end{array}$ & $\begin{array}{l}N \\
W\end{array}$ & 15. 9. & $125-135$ & $\frac{2,65-}{3,65}$ & $\begin{array}{r}33,73- \\
33,51\end{array}$ \\
\hline
\end{tabular}

Soweit gleichzeitig hydrographische Messungen gemacht wurden, besorgte mir Herr Prof. Kalle, Hamburg, liebenswürdigerweise die Angaben über Temperatur und Salzgehalt. Da von 2620 keine Angaben vorliegen, wurden die Meßergebnisse von zwei sehr benachbarten Stationen mit entsprechender Tiefe eingetragen. 
branchiermuskulatur, dementsprechend zeigten auch die Harnstofflecken auf den Papierchromatogrammen der Elasmobranchier sehr große Ausmaße. Shewan (1953) beobachtete erhebliche Mengen von Betain bei den von ihm untersuchten Knorpelfischen. Konzentrationsangaben liegen nicht vor.

Die Stickstoffmengen beim Katzenhai liegen höher als die von RANKE (1959) beschriebenen Werte, doch kann dies auf jahreszeitliche oder standortbedingte Unterschiede zurückzuführen sein. Dasselbe gilt für $\beta$-Alanin.

Auf Grund der papierchromatographischen Analysen (Tabellen 3, 4) unterscheiden sich die Elasmobranchier von den meisten Knochenfischen durch ein stärkeres Vorkommen von Lysin, $\beta$-Alanin und Harnstoff und durch das

Tabelle 2

Stickstoffgehalt in den Muskelextrakten

Art

Chimäre, Chimaera monstrosa L.

Nagelrochen, Raja clavata $L$.

Gefleckter Rochen, Raja montagui Fowler

Glattrochen, Raja batis L.

Katzenhai, Scyliorhinus canicula L.

Dornhai, Squalus acanthias L.

Hundshai, Galeorhinus galeus L.

Tellina equina (Heterodonta)

Neptunia antiqua (Neogastropoda)

Pandalus borealis (Decapoda)

Gonatus Fabricii (Cephalopoda)

$$
\text { Ringmuskel }
$$

Tentakel

Station mg GN mg AN AN $\%$ GN Rest-N in $100 \mathrm{~g} \mathrm{~F}$ in $100 \mathrm{~g} \mathrm{~F}$

$\begin{array}{rrrrr}2301 & 775 & 72 & 9,3 & 703 \\ 2233 & 916 & 161 & 17,6 & 755 \\ 2478 & 1034 & 138 & 12,6 & 896 \\ 2620 & 831 & 234 & 27,7 & 597 \\ 2234 & 1240 & 477 & 36,3 & 763 \\ 2477 & 988 & 149 & 14,8 & 839 \\ 2233 & 904 & 102 & 11,2 & 802 \\ 2477 & 946 & 143 & 14,8 & 803 \\ 2477 & 781 & 74 & 9,1 & 707 \\ & & & & \\ 2478 & & & & \\ 2523 & & & 32,9 & \\ 2526 & & & 51,6 & \\ 2558 & & & & \\ & 509 & 130 & 25,2 & 379 \\ & 438 & 147 & 32,7 & 271\end{array}$

Abkürzungen:

$\mathrm{mg}$ GN in $100 \mathrm{~g} \mathrm{~F}=\mathrm{mg}$ Gesamtstickstoff in $100 \mathrm{~g}$ Frischgewicht

$\mathrm{mg} \mathrm{AN}$ in $100 \mathrm{~g} \mathrm{~F}=\mathrm{mg}$ Aminostickstoff in $100 \mathrm{~g}$ Frischgewicht

AN\%GN $=$ Anteil des Aminostickstoffs in \% am Gesamtstickstoff

Rest-N

$=$ Reststickstoff

Fehlen von Anserin (Carnosin). Weitere Unterschiede zwischen Knorpel- und Knochenfischen bestehen nach SHEWAN (1953) im stärkeren Auftreten von Trimethylaminoxyd, Betain und Sarcosin bei ersteren. Bei einigen Elasmobranchiern wurde Arginin in starken Konzentrationen gefunden; der gleichlautende Befund von Ranke beim Katzenhai wird damit bestätigt. Die Muskulatur der meisten Knochenfische enthält dagegen nur wenig freies Arginin. Auffällig sind auch die meist hohen Konzentrationen von Prolin sowie das häufige Vorkommen der unbekannten Substanzen „13“ und „14“.

Die Rochen unterscheiden sich von den Haien und von der Chimäre durch höheren Gehalt an Aminostickstoff und meist extrem hohes Vorkommen von $\beta$-Alanin. Bei den Haien ist dagegen der Prolingehalt höher, es fehlen aber $\gamma$-Aminobuttersäure und einige unbekannte Substanzen. Leucin/Isoleucin sind bei den Haien nur sehr schwach nachzuweisen.

Individuen derselben Art zeigen Unterschiede im Gehalt an einigen 


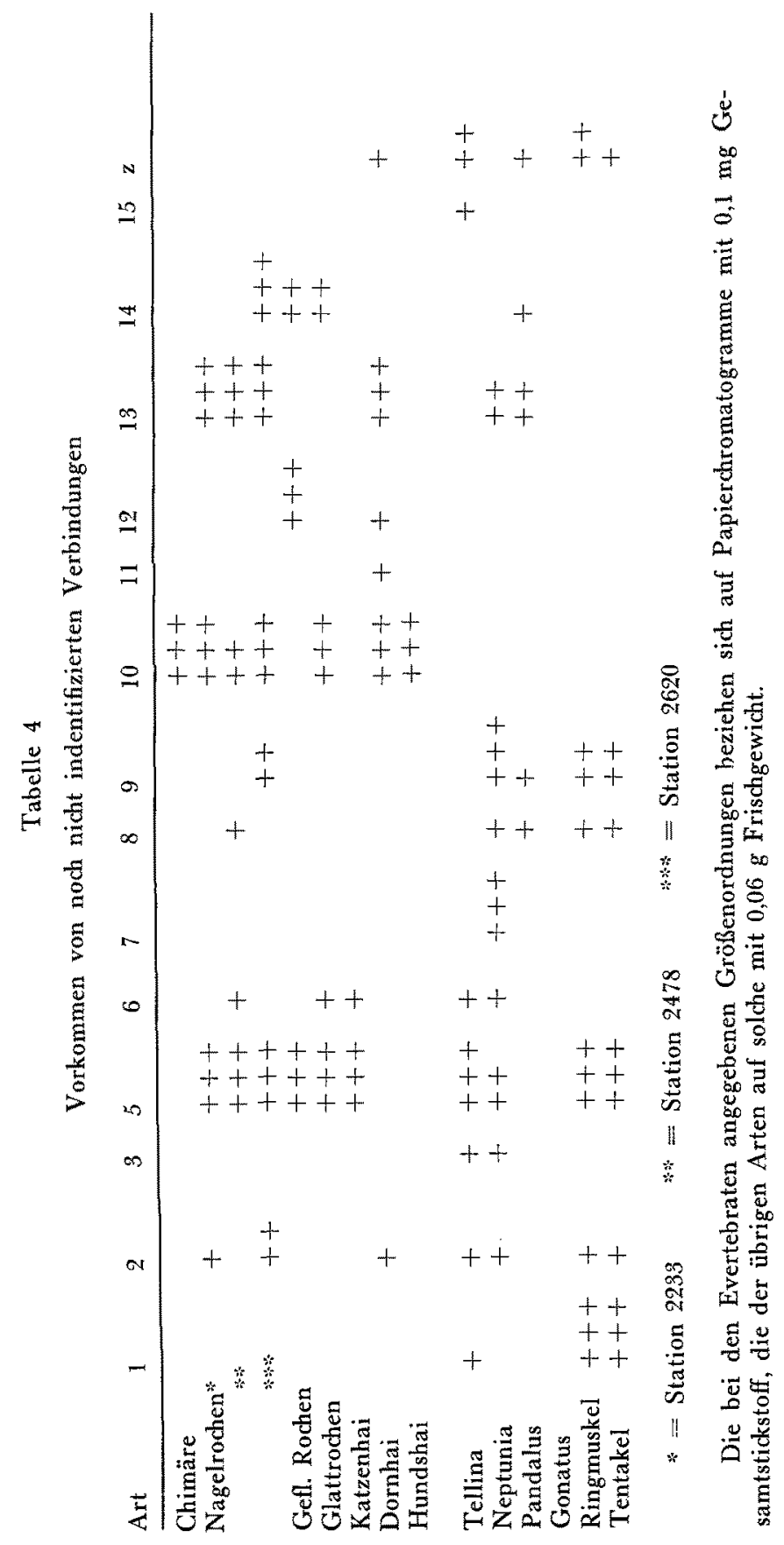




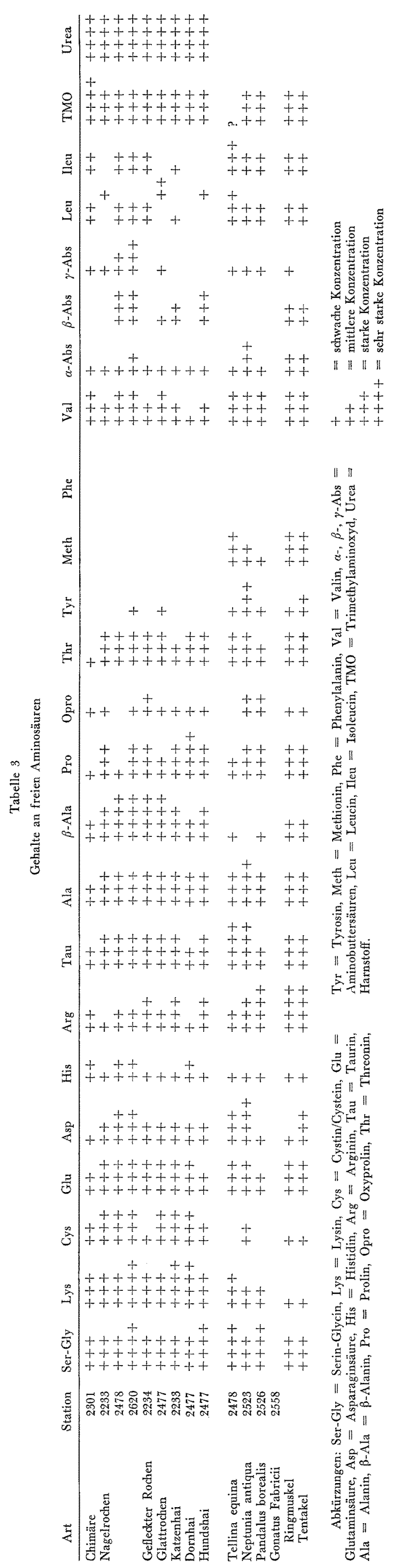


Aminoșäuren, die möglicherweise standortbedingt sind. Ahnliche Beobachtungen konnten bei mehreren Fischarten gemacht werden (SchaEfer 1962).

Die Chimäre nimmt auf Grund ihres niedrigen Gehaltes an Aminostickstoff und der meist geringen Mengen der einzelnen freien Aminosäuren eine Sonderstellung ein.

Die untersuchten Evertebraten weichen stark von den Fischen ab. Die meisten freien Aminosäuren kommen in starken Mengen vor. Auffallend ist, im Gegensatz zur Fischmuskulatur, der meist sehr niedrige Gehalt an Cystin/ Cystein, Lysin und Histidin und das meist starke Vorkommen von Arginin, Prolin, Asparaginsäure und Methionin. Bei einigen anderen Evertebraten wurden gleichfalls große Mengen von Asparaginsäure und Prolin (RANkE) und Arginin (RANke, Simpson et al.) gefunden. Die bei vielen Fischen vorhandenen unbekannten Substanzen „10“, „11" und „12“ sind nicht nachweisbar, statt dessen treten weitere noch nicht identifizierte Flecken auf. RANKE fand auf den Papierchromatogrammen mehrerer Wirbelloser 7 (die identisch mit den Flecken „1“, „2“, „3“, „5“ und „, ${ }^{\text {“ }}$ sein dürften, die beiden übrigen Verbindungen wurden von uns nicht gefunden) und Morrill (1960) 17 Flecken von solchen noch nicht identifizierten Verbindungen. Das Vorkommen solcher Substanzen, die nicht identisch sind mit den bekannten Aminosäuren, dürfte für die qualitative Unterscheidung der Arten von größerem Nutzen sein als das der bekannten Eiweißbausteine.

Eine Diskussion der Ergebnisse, insbesondere der der Evertebraten, erscheint noch verfrüht zu sein. Es muß noch ein großes Artenmaterial untersucht werden, um feststellen zu können, inwieweit das Vorkommen der freien Aminosäuren systematisch verwertbar ist.

Physiologisch wird den niedermolekularen stickstoffhaltigen Substanzen eine große Bedeutung zugemessen. Harnstoff und Trimethylaminoxyd dienen u. a. bei den Elasmobranchiern der Aufrechterhaltung des osmotischen Drucks (Sмiтh 1936). Auch den freien Aminosäuren soll bei den marinen Tieren eine osmoregulatorische Funktion zukommen (Florkin 1956, Simpson et al. 1959, Allen 1961, Jeuniaux et al. 1961).

\section{Zusammenfassung}

Das Muster der freien Aminosäuren und verwandter Verbindungen in der Muskulatur von Chimaera sowie von Elasmobranchiern und marinen Evertebraten wurde papierchromatographisch untersucht.

Der Gehalt an Gesamtstickstoff und Reststickstoff ist bei den Elasmobranchiern bedeutend höher als bei den Knochenfischen.

Die Elasmobranchier unterscheiden sich von den Knochenfischen durch stärkeres Vorkommen einiger freier Aminosäuren und durch das Fehlen von Anserin.

Die Konzentration von $\beta$-Alanin ist bei den Rochen meist extrem hoch. Die Haie enthalten aber meist mehr freies Prolin, es fehlen ihnen $\gamma$-Aminobuttersäure und einige noch nicht indentifizierte Verbindungen.

Die Muskulatur von Chimaera enthält wenig Aminostickstoff und nur geringe Mengen der meisten freien Aminosäuren.

Bei den untersuchten Evertebraten kommen sehr erhebliche Mengen an freien Aminosäuren vor. Cystin/Cystein, Lysin und Histidin sind jedoch nur schwach nachweisbar. Diese Evertebraten unterscheiden sich von den Fischen 
durch stärkeres Auftreten von Arginin, Asparaginsäure, Methionin und Prolin und einiger unbekannter Verbindungen.

\section{Summary}

The pattern of the free amino acids and related compounds in the alcoholic extracts of the muscles of Chimaera and of some elasmobranchs and marine invertebrates has been studied by means of paper chromatography.

The concentrations of total nitrogen and residual nitrogen show greater predominance in the elasmobranchs than in teleosts.

The elasmobranchs differ from the teleosts by higher concentrations of some amino acids and by the absence of anserine.

The quantities of $\beta$-alanine are extremely large in most of the skates. The dogfishes contain more proline, but $\gamma$-amino butyric acid and some unidentified substances could not be detected.

Chimaera contains only small amounts of $\alpha$-amino nitrogen and of most of the amino acids.

Very large quantities of many free amino acids are found in some marine invertebrates. They differ from the fishes by the predominance of arginine, aspartic acid, proline and methionine and of some yet unknown compounds.

Für die Anregung der Arbeit und freundliche Unterstützung danke ich Herrn Prof. Dr. Bramstedt, Hamburg. Die Vorarbeiten konnten dank der gütigen Erlaubnis von Herrn Prof. Dr. Kühnau im Physiologisch-Chemischen Institut, Hamburg, erfolgen. Die Arbeit wurde in der Fischereibiologischen Abteilung des Instituts für Meereskunde, Kiel, und in der Biologischen Anstalt Helgoland durchgeführt. Den Herren Institutsdirektoren danke ich herzlich für die Bereitstellung eines Arbeitsplatzes. Besonderer Dank gebührt den Herren Prof. Dr. Bückmann, Prof. Dr. Kändler und Dr. Aurich für vielfache Unterstützung.

\section{Literaturverzeichnis}

A $1 \mathrm{l}$ e n, K., 1961: The effect of salinity on the amino acid concentration in Rangia cuneata (pelecypoda). Biol. Bull. 121, 419-424.

Florkin, M., 1956: Vergleichende Betrachtung des stationären Zustandes der nicht-eiweißgebundenen Aminosäuren der Tiere. 6. Colloq. Ges. Physiol. Chemie, 62-94. SpringerVerlag, Berlin.

Je uniaux, Ch., Du chât ea u - B o s s on, Ch., and F 1 orkin, M., 1961: Free aminoacids in the intracellular osmoregulation of euryhaline worms (Symposium). Biochem. J. $79,24 \mathrm{p}-25 \mathrm{p}$

Morri11, J. B. Jr., 1960: Concerning the Free Amino Acids in the Hydroid Tubularia. Experientia, 16, 441.

R a n ke, B., 1959: Über die nicht-eiweißgebundenen und eiweißgebundenen Aminosäurenbestände von Fischen, Mollusken und Krebsen. Arch. Fischereiwiss. 10, 117-159.

Schaefer, H., 1962: Freie Aminosäuren und verwandte Verbindungen in der Rumpfmuskulatur frischgefangener mariner Knochenfische. Helgol. Wiss. Meeresunters, 8 $257-275$.

S h e w a n, J. M., 1953: The nitrogenous extractives from fresh fish muscle. II. Comparison of several gadoid and elasmobranch species. J. Sci. Food Agric. 4, 565-568. 
S impson, J. W., A $11 \mathrm{en}, \mathrm{K}$. , and A w a para, J., 1959: Free amino acids in some aquatic invertebrates. Biol. Bull. 117, 371-381.

$\mathrm{S} \mathrm{mith}, \mathrm{H}$. W., 1936: The retention and physiological role of urea in the Elasmobranchii. Biol. Revs. 11, 49-82.

Städeler, G., und Frérichs, F. Th., 1858: Über das Vorkommen von Harnstoff, Taurin und Scyllit in den Organen der Plagiostomen. J. prakt. Chemie 73, 48-55. 